\title{
Global Constraints on Lepton-Quark Contact Interactions
}

\author{
Jorge de Blas $^{a}$, Mikael Chala ${ }^{b}$ and José Santiago ${ }^{b}$ \\ ${ }^{a}$ Department of Physics, University of Notre Dame, \\ Notre Dame, IN 46556, USA \\ ${ }^{b}$ CAFPE and Departamento de Física Teórica y del Cosmos, \\ Universidad de Granada, E-18071 Granada, Spain
}

\begin{abstract}
The Large Hadron Collider can do precision physics at a level that is competitive with electroweak precision constraints when probing physics beyond the Standard Model. We present a simple yet general parameterization of the effect of an arbitrary number of lepton-quark contact interactions on any di-lepton observable at hadron colliders. This parameterization can be easily adopted by the experimental collaborations to put bounds on arbitrary combinations of leptonquark contact interactions. We compute the corresponding bounds from current di-lepton resonance searches at the LHC and find that they are competitive with and often complementary to indirect constraints from electroweak precision data. We combine all current constraints in a global analysis to obtain the most stringent bounds on lepton-quark contact interactions. We also show that the high-energy phase of the LHC has a unique potential in terms of discovery and discrimination power among different types of lepton-quark contact interactions.
\end{abstract}

The Large Hadron Collider (LHC) is a discovery machine at the energy frontier. However, it can also do precision physics, probing physics beyond the Standard Model (SM) in a complementary and even competitive way to electroweak precision data (EWPD). Model-independent bounds on departures from the SM can be systematically computed by means of effective Lagrangians. Assuming the SM particle content and symmetries and neglecting lepton number violation, the leading corrections arise from dimension-six operators

$$
\mathcal{L}_{\text {eff }}=\mathcal{L}_{\mathrm{SM}}+\sum_{i} \frac{\alpha_{i}}{\Lambda^{2}} \mathcal{O}_{i}+\ldots,
$$

where $\mathcal{L}_{\mathrm{SM}}$ is the SM Lagrangian and $\Lambda$ is the cut-off scale at which the effective Lagrangian ceases to be valid. The list of required operators was systematically classified for the first time by Ref. [1]. Several redundancies were discussed by many authors 22 and the first list of truly independent operators was given in Ref. [3] (see Ref. [4 for a recent alternative). In this work we consider the constraints that current searches for di-lepton resonances imply on lepton-quark four-fermion interactions (see Refs. [5, 6] for related analyses). The most general lepton-quark four-fermion interaction can be parameterized in terms of the following dimension-six operators:

$$
\begin{aligned}
\mathcal{O}_{l q}^{(1)}=\left(\bar{l} \gamma^{\mu} l\right)\left(\bar{q} \gamma_{\mu} q\right), & \mathcal{O}_{l q}^{(3)}=\left(\bar{l} \sigma_{I} \gamma^{\mu} l\right)\left(\bar{q} \sigma_{I} \gamma_{\mu} q\right), \\
\mathcal{O}_{e u}=\left(\bar{e} \gamma^{\mu} e\right)\left(\bar{u} \gamma_{\mu} u\right), & \mathcal{O}_{e d}=\left(\bar{e} \gamma^{\mu} e\right)\left(\bar{d} \gamma_{\mu} d\right), \\
\mathcal{O}_{l u}=\left(\bar{l} \gamma^{\mu} l\right)\left(\bar{u} \gamma_{\mu} u\right), & \mathcal{O}_{l d}=\left(\bar{l} \gamma^{\mu} l\right)\left(\bar{d} \gamma_{\mu} d\right), \\
\mathcal{O}_{q e}=\left(\bar{q} \gamma^{\mu} q\right)\left(\bar{e} \gamma_{\mu} e\right), & \mathcal{O}_{q d e}=(\bar{l} e)(\bar{d} q), \\
\mathcal{O}_{l q \epsilon}=(\bar{l} e) \epsilon\left(\bar{q}^{T} u\right), & \mathcal{O}_{q l \epsilon}=(\bar{q} e) \epsilon\left(\bar{l}^{T} u\right),
\end{aligned}
$$


where $l$ and $q$ are the SM lepton and quark doublets; $e, u$ and $d$ denote the SM lepton and quark singlets; $\sigma_{I}$ are the Pauli matrices; and $\epsilon=\mathrm{i} \sigma_{2}$. Flavor indices are not explicitly shown.

In the following we will assume that the operator coefficients are flavor-diagonal and familyuniversal in the quark sector. This guarantees the absence of contributions to quark flavor changing neutral currents (FCNC) from the first seven operators $\left(\mathcal{O}_{l q}^{(1)}-\mathcal{O}_{q e}\right)$. Note that, while EWPD is sensitive to the couplings to all families, the largest constraints from LHC searches come from the coupling to valence quarks. Therefore, we will only consider the contributions to LHC observables from the couplings to the first-generation quarks. Regarding the lepton sector, we consider three different options: a flavor-diagonal family-universal coupling; interactions aligned with the SM fermion basis in a way that there are couplings only to electrons; and couplings only to muons.

For the last three operators $\left(\mathcal{O}_{q d e}, \mathcal{O}_{l q \epsilon}, \mathcal{O}_{q l \epsilon}\right)$ the above assumptions still result on minimal flavor violating interactions in the quark sector. (Coefficients proportional to the quark mixing matrices would be required to remove FCNC.) Even in the absence of FCNC, these three operators still give sizable contributions to charged-current interactions mediating rare decays. Such contributions are strongly constrained, e.g., the experimental value of $R_{\pi}=\Gamma\left(\pi^{+} \rightarrow \nu e^{+}\right) / \Gamma\left(\pi^{+} \rightarrow \nu \mu^{+}\right)$sets bounds $\left|\alpha_{i}\right| / \Lambda^{2} \lesssim \mathcal{O}\left(10^{-3}\right) \mathrm{TeV}^{-2}$ for corrections only to the muon channel, and two orders of magnitude smaller for the electron channel [6]. As we will see, such limits are significantly stronger than the LHC and EWPD constraints discussed here. Therefore, we will not include these three operators in our numerical analyses, although they are included in all our equations for the sake of completeness.

The contribution of the operators in (2) to Drell-Yan production reads, at the partonic level,

$$
\begin{aligned}
48 \pi \frac{d \sigma}{d \hat{t}}\left(\bar{u} u \rightarrow \ell^{+} \ell^{-}\right) & =\left[\left|\mathcal{A}_{u_{L} \ell_{R}}^{\mathrm{SM}}+\frac{\alpha_{q e}}{\Lambda^{2}}\right|^{2}+\left|\mathcal{A}_{u_{R} \ell_{L}}^{\mathrm{SM}}+\frac{\alpha_{l u}}{\Lambda^{2}}\right|^{2}+\frac{1}{2 \Lambda^{4}}\left[\left|\alpha_{q l \epsilon}\right|^{2}+\operatorname{Re}\left(\alpha_{l q \epsilon} \alpha_{q l \epsilon}^{*}\right)\right]\right] \frac{\hat{t}^{2}}{\hat{s}^{2}} \\
& +\left[\left|\mathcal{A}_{u_{L} \ell_{L}}^{\mathrm{SM}}+\frac{\alpha_{l q}^{(1)}-\alpha_{l q}^{(3)}}{\Lambda^{2}}\right|^{2}+\left|\mathcal{A}_{u_{R} \ell_{R}}^{\mathrm{SM}}+\frac{\alpha_{e u}}{\Lambda^{2}}\right|^{2}-\frac{1}{2 \Lambda^{4}} \operatorname{Re}\left(\alpha_{l q \epsilon} \alpha_{q l \epsilon}^{*}\right)\right] \frac{\hat{u}^{2}}{\hat{s}^{2}} \\
& +\frac{1}{2 \Lambda^{4}}\left[\left|\alpha_{l q \epsilon}\right|^{2}+\operatorname{Re}\left(\alpha_{l q \epsilon} \alpha_{q l \epsilon}^{*}\right)\right], \\
48 \pi \frac{d \sigma}{d \hat{t}}\left(\bar{d} d \rightarrow \ell^{+} \ell^{-}\right) & =\left[\left|\mathcal{A}_{d_{L} \ell_{R}}^{\mathrm{SM}}+\frac{\alpha_{q e}}{\Lambda^{2}}\right|^{2}+\left|\mathcal{A}_{d_{R} \ell_{L}}^{\mathrm{SM}}+\frac{\alpha_{l d}}{\Lambda^{2}}\right|^{2}\right] \frac{\hat{t}^{2}}{\hat{s}^{2}} \\
& +\left[\left|\mathcal{A}_{d_{L} \ell_{L}}^{\mathrm{SM}}+\frac{\alpha_{l q}^{(1)}+\alpha_{l q}^{(3)}}{\Lambda^{2}}\right|^{2}+\left|\mathcal{A}_{d_{R} \ell_{R}}^{\mathrm{SM}}+\frac{\alpha_{e d}}{\Lambda^{2}}\right|^{2}\right] \frac{\hat{u}^{2}}{\hat{s}^{2}}+\frac{\left|\alpha_{q d e}\right|^{2}}{2 \Lambda^{4}},
\end{aligned}
$$

where we have defined

$$
\mathcal{A}_{\psi \phi}^{\mathrm{SM}}=\frac{e^{2} Q_{\psi} Q_{\phi}}{\hat{s}}+\frac{g_{\psi} g_{\phi}}{\hat{s}-m_{Z}^{2}+\mathrm{i} m_{Z} \Gamma_{Z}},
$$

with $g_{\psi}=\frac{g}{c_{W}}\left[T_{\psi}^{3}-s_{W}^{2} Q_{\psi}\right], Q$ the electric charge, $T^{3}$ the third component of weak isospin, $g$ the $S U(2)_{L}$ coupling, $m_{Z}$ and $\Gamma_{Z}$ the $Z$-boson mass and width, and $s_{W}\left(c_{W}\right)$ the sine (cosine) of the weak angle. This result completes previous partial calculations [5, 7].

Eqs. (3) allow us to parameterize any di-lepton observable at the LHC in the presence of arbitrary lepton-quark contact interactions. Any such observable can be written in terms of the cross section in a particular region of phase space as measured by experiments, $\sigma$, which can in turn be written in the form of a master equation with a small number of parameters. In the limit of large di-lepton invariant masses, the master equation takes the following simple form

$$
\sigma=\sigma^{\mathrm{SM}}+\frac{1}{\Lambda^{2}} \sum_{q=u, d}\left[F_{1}^{q} A_{1}^{q}+F_{2}^{q} A_{2}^{q}\right]+\frac{1}{\Lambda^{4}} \sum_{q=u, d}\left[G_{1}^{q} B_{1}^{q}+G_{2}^{q} B_{2}^{q}+G_{3}^{q} B_{3}^{q}\right],
$$


where we have neglected corrections proportional to $m_{Z}^{2} / \hat{s} \ll 1$. The coefficients $A_{1,2}^{u, d}$ and $B_{1,2,3}^{u, d}$ encode the dependence on the four-fermion operators

$$
\begin{aligned}
A_{1}^{u} & =\left[e^{2} Q_{u} Q_{e}+g_{u_{L}} g_{e_{L}}\right]\left(\alpha_{l q}^{(1)}-\alpha_{l q}^{(3)}\right)+\left[e^{2} Q_{u} Q_{e}+g_{u_{R}} g_{e_{R}}\right] \alpha_{e u} \\
A_{2}^{u} & =\left[e^{2} Q_{u} Q_{e}+g_{u_{L}} g_{e_{R}}\right] \alpha_{q e}+\left[e^{2} Q_{u} Q_{e}+g_{u_{R}} g_{e_{L}}\right] \alpha_{l u} \\
A_{1}^{d} & =\left[e^{2} Q_{d} Q_{e}+g_{d_{L}} g_{e_{L}}\right]\left(\alpha_{l q}^{(1)}+\alpha_{l q}^{(3)}\right)+\left[e^{2} Q_{d} Q_{e}+g_{d_{R}} g_{e_{R}}\right] \alpha_{e d} \\
A_{2}^{d} & =\left[e^{2} Q_{d} Q_{e}+g_{d_{L}} g_{e_{R}}\right] \alpha_{q e}+\left[e^{2} Q_{d} Q_{e}+g_{d_{R}} g_{e_{L}}\right] \alpha_{l d} \\
B_{1}^{u} & =4\left(\alpha_{l q}^{(1)}-\alpha_{l q}^{(3)}\right)^{2}+4 \alpha_{e u}^{2}-2 \operatorname{Re}\left(\alpha_{l q \epsilon} \alpha_{q l \epsilon}^{*}\right) \\
B_{2}^{u} & =4 \alpha_{q e}^{2}+4 \alpha_{l u}^{2}+2\left|\alpha_{q l \epsilon}\right|^{2}+2 \operatorname{Re}\left(\alpha_{l q \epsilon} \alpha_{q l \epsilon}^{*}\right) \\
B_{3}^{u} & =2\left|\alpha_{l q \epsilon}\right|^{2}+2 \operatorname{Re}\left(\alpha_{l q \epsilon} \alpha_{q l \epsilon}^{*}\right) \\
B_{1}^{d} & =4\left(\alpha_{l q}^{(1)}+\alpha_{l q}^{(3)}\right)^{2}+4 \alpha_{e d}^{2} \\
B_{2}^{d} & =4 \alpha_{q e}^{2}+4 \alpha_{l d}^{2} \\
B_{3}^{d} & =2\left|\alpha_{q d e}\right|^{2} .
\end{aligned}
$$

$\sigma^{\mathrm{SM}}, F_{1,2}^{u, d}$ and $G_{1,2,3}^{u, d}$ on the other hand depend on the particular phase space region we are considering for the observable we want to compute, and encode the effects of the parton distribution functions and the cuts involved in the experimental analyses. A further simplification can be obtained in the case of forward-backward symmetric observables, for which we can impose the following extra conditions:

$$
\left.\begin{array}{ll}
F_{1}^{u}=F_{2}^{u}, & F_{1}^{d}=F_{2}^{d} \\
G_{1}^{u}=G_{2}^{u}, & G_{1}^{d}=G_{2}^{d}
\end{array}\right\} \text { (symmetric observables), }
$$

since the corresponding contributions are related by a $\hat{t} \leftrightarrow \hat{u}$ exchange. Finally, for symmetric observables for which the experimental acceptance is approximately constant along the detector coverage, we can also impose

$$
G_{3}^{u}=3 G_{1}^{u}, \quad G_{3}^{d}=3 G_{1}^{d},
$$

relating the contributions proportional to $\hat{s}$ with those proportional to $\hat{t}$ and $\hat{u}$.

This master equation, Eqs. (4) and (5), constitutes the main result of the present paper. It can be easily adopted by the LHC experimental collaborations and, once they have computed the specific values of the observable-dependent coefficients and their uncertainties, bounds on arbitrary combinations of lepton-quark contact interactions can be easily obtained.

To show how this can be done, we have computed the observable-dependent coefficients by implementing the effective operators in FeynRules 1.6 [8]. We have then used MadGraph 5 [9] to generate di-lepton events at the partonic level, Pythia 6 [10] for hadronization and showering and Delphes 3.0.9 [11] for fast detector simulation. We have implemented the latest ATLAS [12] and CMS [13] di-lepton searches using all the collected luminosity at the LHC with $\sqrt{s}=8 \mathrm{TeV}$. Di-electron and dimuon final states are studied separately in these analyses. We have considered results for the following bins in the di-lepton invariant mass (in $\mathrm{TeV}$ ) to avoid contamination from non-Drell Yan backgrounds

$$
\begin{array}{rll}
\text { CMS } & : & b_{1}=[0.9,1.3], \quad b_{2}=[1.3,1.8], \quad b_{3}=[1.8,-], \\
\text { ATLAS } & : & b_{4}=[1.2,3],
\end{array}
$$

resulting in a total of 8 bins, counting electrons and muons. The observable considered is the number of events on each bin. This is a symmetric observable for which the experimental acceptances are reasonably constant along the detector coverage. Thus we can use the simplifying conditions Eqs. (6) 


\begin{tabular}{ccccccccc}
\hline & $b_{1}(e)$ & $b_{2}(e)$ & $b_{3}(e)$ & $b_{4}(e)$ & $b_{1}(\mu)$ & $b_{2}(\mu)$ & $b_{3}(\mu)$ & $b_{4}(\mu)$ \\
\hline$N_{\mathrm{SM}}$ & 32.6 & 4.68 & 0.60 & 8.72 & 37.0 & 5.38 & 0.74 & 9.44 \\
$F_{1}^{u}$ & 2514 & 731 & 202 & 1324 & 2746 & 811 & 251 & 1410 \\
$F_{1}^{d}$ & 1484 & 359 & 80.2 & 677 & 1590 & 481 & 93.6 & 775 \\
$G_{1}^{u}$ & 346 & 203 & 116 & 404 & 376 & 219 & 134 & 415 \\
$G_{1}^{d}$ & 200 & 106 & 46.1 & 199 & 219 & 118 & 53.0 & 207 \\
\hline$N_{\text {Obs }}$ & 41 & 4 & 0 & 10 & 49 & 11 & 1 & 8 \\
\hline
\end{tabular}

Table 1: Observable-dependent coefficients for di-lepton LHC searches (see Eq. (8) for details). The observable considered is the number of events on each bin. The coefficients $F_{1}^{u, d}$ and $G_{1}^{u, d}$ are in units of $\mathrm{TeV}^{-2}$ and $\mathrm{TeV}^{-4}$, respectively. Eqs. (6) and (7) should be used to fix the remaining parameters. The observed number of events is also reported in each case.

and (7). We have checked that these simplifying conditions lead to the correct number of events within a $3 \%$ uncertainty. The corresponding parameters for our master equation are reported in Table 1, together with the actual number of observed events. Note that only a small number of simulations are required to obtain these parameters. This is an important advantage for experimental collaborations that rely on expensive full detector simulations. In particular they would have to compute the expected number of events in the SM (separately for signal and background) plus the expected number of events in the presence of just two operators, that can be taken for instance $\mathcal{O}_{e u}$ and $\mathcal{O}_{e d}$, for two values of the corresponding coefficients. This would suffice to fully generate our master equation for an arbitrary combination of lepton-quark contact interactions. We have implemented all the operators and extensively tested the validity of our master equation and the approximations in Eqs. (6) and (7).

Once we have the prediction for the number of events in each bin and experiment for arbitrary combinations of quark-lepton contact interactions we can obtain the corresponding bounds on the coefficients of such operators. For the sake of concreteness, we consider in the following that only one operator is present at a time. We use the $\mathrm{CL}_{s}$ method [14] to obtain the $95 \%$ confidence level (C.L.) bound on the coefficients of the different operators. The combination of different bins is performed by defining an effective $\chi^{2}$ function for each bin,

$$
\chi^{2}=2\left[\operatorname{Erf}^{-1}\left(1-\mathrm{CL}_{s}\right)\right]^{2},
$$

and adding the $\chi^{2}$ of all bins. This allows a direct combination with constraints from EWPD and is exact in the limit of a large number of events. Given the fact that we have several bins with a small number of events, we have tested the validity of such an approximation by computing the probability density function of the combined $\mathrm{CL}_{s}$ test statistic by Monte Carlo simulation using the TLimits Root class. The resulting bounds agree with our approximation within $10 \%$ on average, but they depart by about $20 \%$ in some cases. To account for this uncertainty, we have imposed a $20 \%$ penalty on the coefficients of the effective operators when computing the LHC $\chi^{2}$. This means that the coefficients of the different effective operators are multiplied by a 0.8 factor before inserting them in the calculation of the LHC $\chi^{2}$. With this penalty the bounds obtained with the $\chi^{2}$ are always conservative as compared with the exact bounds computed with the Monte Carlo method. We present the corresponding bounds in columns $2-4$ of Table 2 for the three different flavor options mentioned in the introduction. The limits range from 0.02 to $0.13 \mathrm{TeV}^{-2}$. This also applies to the last three operators in (2), and thus justifies neglecting them in our analysis, since the $R_{\pi}$ constraints make their effects invisible at the LHC at $\sqrt{s}=8 \mathrm{TeV}$. 


\begin{tabular}{ccccccc}
\hline \multicolumn{8}{c}{$95 \%$ C.L. limits on $\frac{\alpha_{i}}{\Lambda^{2}}\left[\mathrm{TeV}^{-2}\right]$} \\
$\mathcal{O}_{i}$ & Universal & Only $e$ & Only $\mu$ & Universal & Only $e$ & Only $\mu$ \\
\hline $\mathcal{O}_{l q}^{(1)}$ & {$[-0.032,0.073]$} & {$[-0.040,0.082]$} & {$[-0.043,0.084]$} & {$[-0.012,0.055]$} & {$[-0.012,0.055]$} & {$[-0.620,0.669]$} \\
$\mathcal{O}_{l q}^{(3)}$ & {$[-0.106,0.019]$} & {$[-0.118,0.026]$} & {$[-0.126,0.026]$} & {$[-0.006,0.012]$} & {$[-0.006,0.012]$} & {$[-0.169,0.694]$} \\
$\mathcal{O}_{e u}$ & {$[-0.032,0.102]$} & {$[-0.042,0.113]$} & {$[-0.044,0.117]$} & {$[-0.097,0.017]$} & {$[-0.097,0.017]$} & - \\
$\mathcal{O}_{e d}$ & {$[-0.107,0.068]$} & {$[-0.123,0.084]$} & {$[-0.128,0.086]$} & {$[-0.077,0.040]$} & {$[-0.077,0.040]$} & - \\
$\mathcal{O}_{l u}$ & {$[-0.043,0.079]$} & {$[-0.054,0.090]$} & {$[-0.056,0.093]$} & {$[-0.041,0.095]$} & {$[-0.045,0.092]$} & {$[-0.335,0.889]$} \\
$\mathcal{O}_{l d}$ & {$[-0.096,0.076]$} & {$[-0.112,0.093]$} & {$[-0.117,0.095]$} & {$[-0.021,0.106]$} & {$[-0.020,0.107]$} & {$[-1.337,1.407]$} \\
$\mathcal{O}_{q e}$ & {$[-0.040,0.058]$} & {$[-0.049,0.068]$} & {$[-0.051,0.070]$} & {$[-0.055,0.011]$} & {$[-0.055,0.011]$} & - \\
\hline
\end{tabular}

Table 2: Comparison of the different 95\% C.L. limits on lepton-quark contact interactions. The three different flavor realizations discussed in the introduction are denoted "Universal", "Only $e$ " and "Only $\mu$ ", respectively. In all cases we assume diagonal and family-universal interactions with quarks. A dash ("-") is used to indicate those cases where the data cannot bound the corresponding operator.

Lepton-quark contact interactions also contribute to precision observables, and therefore are indirectly constrained by EWPD. These limits are dominated by low-energy measurements (e.g., atomic parity violation experiments) and by the $e^{+} e^{-} \rightarrow$ hadrons data taken at energies above the $Z$ pole at LEP2. The electroweak bounds for all the dimension-six interactions that can be generated at tree level and can interfere with the SM were computed in Refs. [15, 16]. The EWPD fits in this work include all the updates discussed in the analysis of the electroweak constraints in Ref. [17, the latest values of $\alpha_{S}$ and the top mass, and the final results of $e^{+} e^{-} \rightarrow \bar{f} f$ at LEP2 [18. In all cases, we assume real values for the dimension-six operator coefficients. We extend the results in Ref. [16] for the first seven operators in (2), for the case of diagonal and universal quarks interactions, and the different lepton flavor hypotheses discussed in the introduction ${ }^{1}$ The corresponding bounds are shown in the last three columns of Table 2 ,

Several conclusions can be extracted from the results in Table2, Even though indirect constraints from EWPD are still in many cases more stringent than those from LHC searches, the latter are already quite competitive in general and in some cases much superior. The operators involving muons are very poorly constrained by EWPD. In these cases the LHC constraints are more than an order of magnitude more stringent. The previous most stringent bounds on these operators come from Tevatron data [19]. They are discussed in Ref. [6] and are weaker than the ones from LHC data. Also there is quite often (see, e.g., $\left.\mathcal{O}_{e u, l u, l d, q e}\right)$ a nice complementarity between both results, with each experimental data set (EWPD or LHC) improving the worst limit derived from the other. It should be noted, however, that, due to the different energies probed by each set, the range of validity of the effective description is different in each case. In particular, since we are probing energies up to $\sim 3$ $\mathrm{TeV}$ in LHC searches, the bounds we have obtained are only valid if the coefficients of the effective operators satisfy

$$
\left.\alpha \gtrsim 9 \frac{\alpha}{\Lambda^{2}}\right|_{\max } \text { or }\left.\alpha \lesssim 9 \frac{\alpha}{\Lambda^{2}}\right|_{\min },
$$

where $\alpha /\left.\Lambda^{2}\right|_{\max (\min )}$ stands for the upper bound in the case of a positive (negative) $\alpha$.

Once we have obtained the bounds on lepton-quark contact interactions from LHC searches and

\footnotetext{
${ }^{1}$ In Ref. [16] all fermion interactions are assumed to be diagonal and family-universal. Also, a different basis for four-fermion interactions is employed. In particular, the operators $\mathcal{O}_{l u, l d, q e}$ in Ref. [16] are related to those in this paper by the Fierz reordering $\left(\overline{\psi_{L}^{1}} \gamma^{\mu} \psi_{L}^{2}\right)\left(\overline{\xi_{R}^{3}} \gamma_{\mu} \xi_{R}^{4}\right)=-2\left(\overline{\psi_{L}^{1}} \xi_{R}^{4}\right)\left(\overline{\xi_{R}^{3}} \psi_{L}^{2}\right)$.
} 


\begin{tabular}{|c|c|c|c|}
\hline \multirow[b]{2}{*}{$\mathcal{O}_{i}$} & \multicolumn{3}{|c|}{$95 \%$ C.L. Combined limits on $\frac{\alpha_{i}}{\Lambda^{2}}\left[\mathrm{TeV}^{-2}\right]$} \\
\hline & Universal & Only $e$ & Only $\mu$ \\
\hline $\mathcal{O}_{l q}^{(1)}$ & {$[-0.011,0.053]$} & {$[-0.012,0.053]$} & {$[-0.042,0.084]$} \\
\hline $\mathcal{O}_{l q}^{(3)}$ & {$[-0.006$} & {$[-0.00$} & {$[-0.117,0.027]$} \\
\hline $\mathcal{O}_{e u}^{u}$ & {$[-0.036,0.026]$} & {$[-0.04$} & {$[-0.044,0.117]$} \\
\hline $\mathcal{O}_{e d}$ & {$[-0.07$} & {$[-0.0$} & {$[-0.12$} \\
\hline $\mathcal{O}_{l u}$ & {$[-0.0$} & {$[-0.0$} & {$[-0.05$} \\
\hline $\mathcal{O}_{l d}$ & {$[-0.02$} & {$[-0.02$} & {$[-0.117,0.094]$} \\
\hline $\mathcal{O}_{q e}$ & {$[-0.038,0.013]$} & {$[-0.043,0.012]$} & {$[-0.051,0.070]$} \\
\hline
\end{tabular}

Table 3: Combination of the different 95\% C.L. limits on lepton-quark contact interactions.

EWPD, we can consider the bounds obtained from a joint analysis of both data sets. This global analysis provides the most stringent constraints over the lepton-quark contact interactions under consideration. The combination is performed by adding the effective $\chi^{2}$ in Eq. $(9)$ for all bins from all the LHC searches to the $\chi^{2}$ of the electroweak fit. The $95 \%$ C.L. bounds we have obtained with this procedure are reported in Table 3 .

Given how stringent the global constraints are and the fact that LHC searches with $\sqrt{s}=8 \mathrm{TeV}$ are already competitive with EWPD bounds, it is worth considering the ability of the LHC to measure these operators in di-lepton searches during its high-energy phase. Furthermore, in case a departure from the SM prediction is observed, it would be crucial to try to understand the origin of such a departure. It is clear that any di-lepton search at the LHC can be only sensitive to the combination of operators described by the coefficients in Eq. (5). Nevertheless, a very simple study of angular distributions can discriminate between contributions that are mostly forward, mostly backward or symmetric. In order to test this, we have generated di-muon events at $\sqrt{s}=14 \mathrm{TeV}$ and computed the observed number of events and a forward-backward asymmetry, defined as

$$
A_{F B}=\frac{\sigma(\overline{\Delta \eta}>0)-\sigma(\overline{\Delta \eta}<0)}{\sigma(\overline{\Delta \eta}>0)+\sigma(\overline{\Delta \eta}<0)},
$$

where $\overline{\Delta \eta} \equiv\left(\eta_{l^{-}}-\eta_{l^{+}}\right) /\left(\eta_{l^{-}}+\eta_{l^{+}}\right)$is positive (negative) for a forward (backward) negatively charged lepton in the center of mass frame, with respect to the direction of the incoming quark (when this direction is estimated by the beam axis in the direction of the di-lepton momentum in the lab frame).

We show in Fig. 1 the di-muon $A_{F B}$ as a function of the observed number of events, both computed with di-muon candidates with $M_{\mu^{+} \mu^{-}} \geq 1.8 \mathrm{TeV}$, for two representantive operators, at the LHC with $\sqrt{s}=14 \mathrm{TeV}$ and an integrated luminosity of $300 \mathrm{fb}^{-1}$. As a reference we also plot the expected values for the SM. In the figure we have varied the coefficients of the different operators within their current limits. Both discovery in terms of number of events and discrimination among different operators are clearly possible at this center of mass energy.

In summary, we have provided a general parameterization of the effect of an arbitrary number of lepton-quark contact interactions on di-lepton production at hadron colliders. This is expressed in the form of a master equation, Eqs. (4) and (5), in terms of a small number of observable-dependent parameters (4 plus the SM prediction for forward-backward symmetric observables). Once these parameters have been computed for the particular observable considered, the bounds on an arbitrary combination of lepton-quark contact interactions can be obtained. We have also found that it is important to consider more than one bin in di-lepton invariant masses as different operators are more 


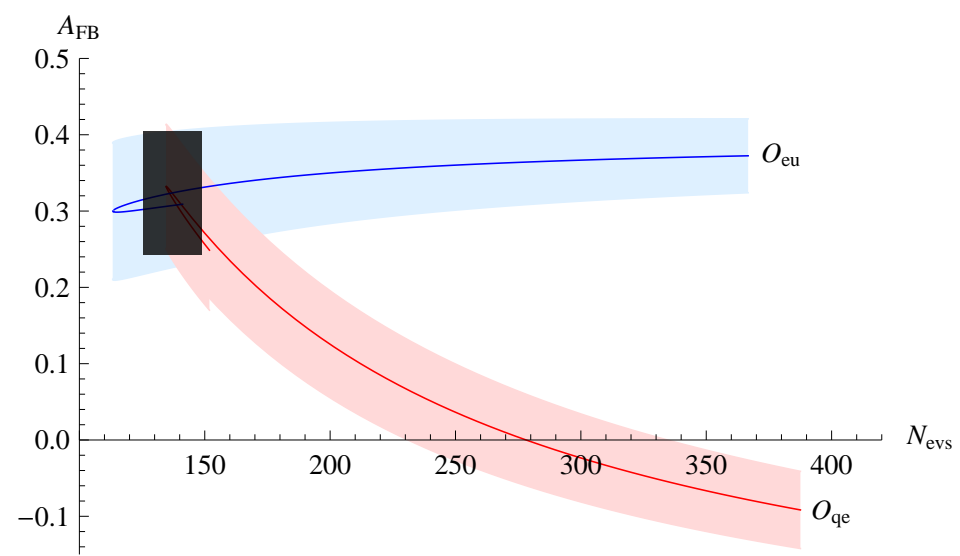

Figure 1: Forward-backward asymmetry as a function of the observed number of events for two representative operators. We have considered $\sqrt{s}=14 \mathrm{TeV}$ with $300 \mathrm{fb}^{-1}$ of integrated luminosity and $M_{\ell^{+} \ell^{-}} \geq 1.8 \mathrm{TeV}$. The coefficients of the operators are varied within the current allowed values. The bands represent the $1 \sigma$ uncertainty on the asymmetry. The SM result is represented, with $1 \sigma$ uncertainties with a gray rectangle.

efficiently constrained at different values of the di-lepton invariant mass. We have shown how to obtain such constraints by combining LHC searches with indirect constraints from EWPD, assuming one operator at a time. LHC searches are already sometimes competitive and quite often complementary to EWPD. The very stringent global constraints that we have obtained still leave room for a discovery at the high-energy phase of the LHC. We have also discussed how one can use angular observables to distinguish among different classes of lepton-quark contact interactions.

\section{Acknowledgments}

We thank F. del Águila for useful comments. The work of J.B. has been supported in part by the U.S. National Science Foundation under Grant PHY-1215979. The work of M.C. and J.S. has been partially supported by MINECO projects FPA2006-05294 and FPA2010-17915, by Junta de Andalucía grants FQM 101 and FQM 6552. M.C. is also supported by the MINECO under the FPU program.

\section{References}

[1] W. Buchmuller and D. Wyler, Nucl. Phys. B 268 (1986) 621.

[2] R. Rattazzi, Z. Phys. C 40 (1988) 605; B. Grzadkowski, Z. Hioki, K. Ohkuma and J. Wudka, Nucl. Phys. B 689 (2004) 108 hep-ph/0310159; P. J. Fox, Z. Ligeti, M. Papucci, G. Perez and M. D. Schwartz, Phys. Rev. D 78 (2008) 054008 [arXiv:0704.1482 [hep-ph]]; J. A. AguilarSaavedra, Nucl. Phys. B 812 (2009) 181 arXiv:0811.3842 [hep-ph]]; Nucl. Phys. B 821 (2009) 215 [arXiv:0904.2387 [hep-ph]]; C. Grojean, W. Skiba and J. Terning, Phys. Rev. D 73 (2006) 075008 [hep-ph/0602154].

[3] B. Grzadkowski, M. Iskrzynski, M. Misiak and J. Rosiek, JHEP 1010 (2010) 085 arXiv:1008.4884 [hep-ph]]. 
[4] R. Contino, M. Ghezzi, C. Grojean, M. Muhlleitner and M. Spira, JHEP 1307 (2013) 035 arXiv:1303.3876 [hep-ph]].

[5] N. Di Bartolomeo and M. Fabbrichesi, Phys. Lett. B 406 (1997) 237 hep-ph/9703375]; A. E. Nelson, Phys. Rev. Lett. 78 (1997) 4159 [hep-ph/9703379]; W. Buchmuller and D. Wyler, Phys. Lett. B 407 (1997) 147 hep-ph/9704317]; V. D. Barger, K. -m. Cheung, K. Hagiwara and D. Zeppenfeld, Phys. Rev. D 57 (1998) 391 hep-ph/9707412]; A. F. Zarnecki, Eur. Phys. J. C 11 (1999) 539 hep-ph/9904334; K. -m. Cheung, Phys. Lett. B 517 (2001) 167 [hep-ph/0106251]; A. Friedland, M. L. Graesser, I. M. Shoemaker and L. Vecchi, Phys. Lett. B 714 (2012) 267 arXiv:1111.5331 [hep-ph]].

[6] M. Carpentier and S. Davidson, Eur. Phys. J. C 70 (2010) 1071 arXiv:1008.0280 [hep-ph]].

[7] E. Eichten, K. D. Lane and M. E. Peskin, Phys. Rev. Lett. 50 (1983) 811, and references there in.

[8] N. D. Christensen and C. Duhr, Comput. Phys. Commun. 180 (2009) 1614 arXiv:0806.4194 [hep-ph]].

[9] J. Alwall, M. Herquet, F. Maltoni, O. Mattelaer and T. Stelzer, JHEP 1106 (2011) 128 arXiv:1106.0522 [hep-ph]].

[10] T. Sjostrand, S. Mrenna and P. Z. Skands, JHEP 0605 (2006) 026 [hep-ph/0603175].

[11] S. Ovyn, X. Rouby and V. Lemaitre, arXiv:0903.2225 [hep-ph].

[12] ATLAS Collaboration, ATLAS-CONF-2013-017.

[13] CMS Collaboration, CMS-PAS-EXO-12-027; CMS-PAS-EXO-12-031.

[14] J. Beringer et al. [Particle Data Group Collaboration], Phys. Rev. D 86 (2012) 010001.

[15] Z. Han and W. Skiba, Phys. Rev. D 71 (2005) 075009 hep-ph/0412166.

[16] F. del Aguila and J. de Blas, Fortsch. Phys. 59 (2011) 1036 arXiv:1105.6103 [hep-ph]].

[17] J. de Blas, J. M. Lizana and M. Perez-Victoria, JHEP 1301 (2013) 166 [arXiv:1211.2229 [hep-ph]].

[18] S. Schael et al. [ALEPH and DELPHI and L3 and OPAL and LEP Electroweak Working Group Collaborations], arXiv:1302.3415 [hep-ex].

[19] http://www-d0.fnal.gov/Run2Physics/WWW/results/np.htm, D0 note 4922-CONF, D0 note 4552- CONF. 